\title{
Resultados Perinatais em Grávidas com mais de 35 Anos: Estudo Controlado
}

\author{
Perinatal Results in Pregnant Women with more than 35 Years: A Controlled Study
}

\begin{abstract}
Priscilla Chamelete Andrade, José Juvenal Linhares, Silvio Martinelli,
\end{abstract} Marcelo Antonini, Umberto Gazi Lippi, Fausto Farah Baracat

\section{RESUM0}

Objetivo: avaliar os resultados perinatais em gestantes com mais de 35 anos e verificar diferenças nos grupos entre 35 e 39 anos e acima de 40 anos.

Métodos: entre janeiro de 2000 e julho de 2003, realizou-se estudo retrospectivo por análise de fichas obstétricas de 3093 gestantes, excluindo-se 933 gestantes. As pacientes foram divididas em 3 grupos: 18 a 29 anos (grupo controle), 30 a 39 anos e mais de 40 anos. A coleta de dados foi realizada por meio de formulário padronizado e os dados foram transferidos para uma planilha eletrônica (Excel-Microsoft Office 2000). Para a análise estatística, foram utilizados o teste do $\chi^{2}$ e o teste de Fisher, sendo considerado risco a (alfa) menor ou igual a $5 \%$ e intervalo de confiança de $95 \%$.

Resultados: a via de parto mais utilizada para essas pacientes foi a cesárea, tanto no grupo de 35 a 39 anos (438/792; 55,3\%), quanto nas gestantes com mais de 40 anos (153/236; $64,8 \%)$. A taxa de prematuridade (39/236; 16,5\%), o baixo peso ao nascer (37/236; 15,7\%) e a restrição de crescimento fetal $(38 / 236 ; 16,1 \%)$ foram mais altas entre as gestantes tardias, com mais de 40 anos, com diferença significante em relação aos demais grupos. Quanto à ocorrência de óbito fetal, foi constatado nas gestantes de 40 anos incidência cinco vezes maior quando comparado aos outros grupos (diferença estatisticamente significante).

Conclusão: a comparação entre o grupo de gestantes de 35 a 39 anos com o grupo controle apenas diferiu significantemente quanto ao índice de cesáreas, o que nos permite sugerir acompanhamento pré-natal diferenciado para o grupo de gestantes acima de 40 anos.

PALAVRAS-CHAVE: Idade materna. Gravidez normal. Resultado perinatal. Prematuridade. Restrição de crescimento fetal.

\section{Introdução}

O Conselho da Federação Internacional de Ginecologia e Obstetrícia definiu, em 1958, como primíparas idosas as pacientes com 35 anos ou

Serviço de Ginecologia e Obstetrícia do Hospital do Servidor Público Estadual "Franscisco Morato de Oliveira" - São Paulo - SP

Correspondência:

Priscilla Chamelete Andrade

Rua Osaka, 691 - Jd. Japão

02124-040 - São Paulo - SP

Telefone: (11) 6987-1347

priandrade@hotmail.com mais. Atualmente, as gestações após 35 anos são denominadas gestações tardias ${ }^{1}$. Na última década, o número de primigestas com mais de 30 anos dobrou e houve aumento de $80 \%$ dos casos para as com mais de 40 anos. No ano 2000, aproximadamente $10 \%$ de todos os nascimentos ocorreram em mulheres com 35 anos ou mais ${ }^{2}$. As gestantes com mais de 45 anos são consideradas de "idade materna muito avançada". Nessas, além das doenças mais incidentes nas gestações tardias (diabete, hipertensão crônica, hipotiroidismo), ganha importância a maior proporção de cromossomopatias $(9,9 \%)$, com prevalência da trissomia do $21^{3-5}$. 
Muitas mulheres estão adiando sua gestação para a quarta ou quinta décadas para priorizar sua carreira, buscando estabilidade financeira e parceiro estável ${ }^{6}$. Recentes avanços nas técnicas de reprodução assistida têm aumentado o sucesso de gravidez nessas pacientes ${ }^{7}$.

Os dados publicados sobre os riscos associados a gestações acima de 35 anos são inconsistentes e conflitantes, devido a outras variáveis de confusão, como paridade e doenças preexistentes, que prejudicam a avaliação de riscos associados com a idade materna isoladamente ${ }^{7}$.

A gestação em mulheres com 35 anos ou mais está associada a risco aumentado para complicações maternas (maior ganho de peso, obesidade, diabete melito, hipertensão arterial crônica, pré-eclâmpsia e miomas), fetais e do recémnascido (anormalidades cromossômicas e abortamentos espontâneos, mecônio intraparto, baixo peso ao nascer, restrição do crescimento fetal (RCF), macrossomia, sofrimento fetal, internação em UTI e óbito neonatal).

Nessas gestantes também ocorre aumento de complicações obstétricas (trabalho de parto prematuro, hemorragia anteparto, trabalho de parto prolongado, gestação múltipla, apresentações anômalas, distocias, placenta prévia, pós-datismo, oligo e polidrâmnio, rotura prematura de membranas e parto cesáreo) ${ }^{8-10}$.

Em contrapartida, outros estudos, na década de 80 , não mostraram diferença significativa na freqüência destas complicações entre gestantes jovens e aquelas com gestação tardia ${ }^{6}$. Ainda hoje, existem dúvidas se uma mulher com mais de 35 anos, gozando de boa saúde, sem história de infertilidade, não fumante e com características sociodemográficas favoráveis apresenta risco gestacional mais elevado.

Para alguns autores, a idade materna avançada é considerada fator de risco independente para o baixo peso ao nascer, trabalho de parto prematuro, placenta prévia e de recém-nascidos que necessitam de cuidados intensivos em $\mathrm{UTI}^{6}$. Nas gestantes tardias ocorre aumento da taxa de abortamentos e de anormalidades cromossômicas ${ }^{11}$, sendo que 40 a $60 \%$ dos abortos são cromossomicamente anormais ${ }^{12}$. As gestantes com 40 anos ou mais têm maior incidência de complicações antenatais, doenças crônicas, induções de trabalho de parto, partos operatórios e de cesárea eletiva ${ }^{3}$.

Na década de 90, Prysac et al. ${ }^{1}$, Yuan et al. ${ }^{13}$ e Dulitzki et al. ${ }^{5}$ mostraram altas taxas de anestesia de condução, indução do trabalho de parto e de partos operatórios em mulheres nulíparas com 35 anos ou mais quando comparadas com as de 25 a 29 anos.
Segundo alguns estudos, a história pregressa de doenças clínicas ou antecedentes cirúrgicos, tratamento de infertilidade, trabalho de parto prematuro, baixo peso ao nascer, apresentações anômalas e complicações obstétricas são mais freqüentes nas primíparas com idade avançada, justificando o maior número de cesáreas, de necessidade de cuidados intensivos neonatais e de menor Apgar de primeiro minuto em tais pacientes ${ }^{14-20}$.

A hemorragia anteparto e a hipertensão são responsáveis pelo aumento das induções de parto, aumentando as chances de sofrimento fetal iatrogênico e de partos operatórios. O número de cesáreas costuma ser maior entre as primíparas do que em multíparas com mais de 40 anos, tendo como principais indicações o sofrimento fetal e as falhas na indução ${ }^{4}$.

A mortalidade materna aumenta nas pacientes com gestação tardia, principalmente por préeclampsia, placenta prévia, hemorragia pós-parto, embolia pulmonar, embolia por líquido amniótico e outras complicações puerperais ${ }^{21}$. Metade das mortes maternas poderiam ser prevenidas pelo acesso a cuidados básicos de saúde, principalmente durante a gestação, parto e puerpério ${ }^{22-24}$.

De acordo com os relatos de Romero Gutiérrez et al. ${ }^{25} \mathrm{e}$ Vercellini et al. ${ }^{26}$, a idade por si só não aumenta as complicações obstétricas pré-natais de mulheres de 40 anos ou mais, não justificando a indicação de cesárea pela idade materna isolada. Além disso, a indicação da cesárea em pacientes com idade avançada, sem outro fator de risco, pode aumentar a morbidade e os custos hospitalares ${ }^{12,20,27}$.

A ansiedade do médico e da paciente quando se deparam com uma gestação muito desejada, em fase de decréscimo da fertilidade e freqüentemente após o uso de técnicas de reprodução assistida, induzem a escolha da via de parto, aumentando o número de cesáreas sem indicação precisa ${ }^{3,9,18}$.

Alguns estudos indicam aumento no risco da mortalidade perinatal, baixo peso ao nascer, parto pré-termo e fetos pequenos para a idade gestacional em gestações tardias ${ }^{9,17,21}$.

As doenças associadas à idade são fatores determinantes para a ocorrência de efeitos adversos nessas pacientes, levando a aumento da mortalidade e morbidade perinatais em conseqüência de prematuridade, insuficiência uteroplacentária e descolamento prematuro de placenta. Isso pode ser explicado por lesões escleróticas em mulheres idosas que tornam as artérias miometriais incapazes de se adaptar ao aumento hemodinâmico da gestação, explicando também os casos de RCF e de baixo peso ao nascer ${ }^{10,13,15}$. 
A idade materna avançada, a nuliparidade e o tabagismo são fatores independentes associados ao risco de óbito perinatal, que aumenta após os $40 \operatorname{anos}^{8,11}$.

Foram poucos os estudos nacionais que abordaram as gestações após os 35 anos. Laurenti e Buchalla $^{24}$, em 1985, demonstraram aumento da mortalidade perinatal em recém-nascidos de baixo peso de mães nos extremos da idade reprodutiva, assim como nas usuárias de mais de 10 cigarros/ dia. Halpern et al. ${ }^{27}$, em 1996, relataram correlação de baixo peso ao nascer e prematuridade com hospitalização materna durante a gravidez em gestantes com 20 anos ou menos, porém sem relato de índices maiores em gestantes tardias. Azevedo et al. ${ }^{23}$, em 2002, verificaram aumento de prematuridade e de baixo peso ao nascer nos extremos da vida reprodutiva. A macrossomia predominou nas gestantes com 35 anos ou mais, não evidenciando aumento no número de cesáreas nos extremos de idade, pelo grande número de partos domiciliares nessa população.

Em vista dos relatos conflitantes de alguns estudos publicados e da escassez de estudos nacionais sobre esse assunto, nos propusemos a avaliar os resultados perinatais em gestantes com mais de 35 anos em nossa população e verificar diferenças de grupos entre 35 e 39 anos e acima de 40 anos.

\section{Pacientes e Métodos}

Foram analisadas as fichas obstétricas de 3093 gestantes que tiveram parto no Hospital do Servidor Público Estadual "Francisco Morato Oliveira" - São Paulo - SP, no período de janeiro de 2000 a julho de 2003. A coleta de dados foi realizada com formulário padronizado e os dados referentes ao estudo foram transferidos para uma planilha eletrônica (Excel - Microsoft Office 2000).

Foram constituídos três grupos de gestantes, de acordo com a idade, para comparação: grupo de 18 a 29 anos (controle), grupo de 35 a 39 anos e grupo com idade superior a 40 anos.

As variáveis estudadas foram: idade materna, idade gestacional ao parto, peso do recém-nascido ao nascimento, tipo de parto, índice de Apgar de $1^{\circ}$ e $5^{\circ}$ minutos e óbitos fetais.

Para o diagnóstico de RCF, utilizamos o peso de nascimento abaixo do $10^{\circ}$ percentil para a idade gestacional, segundo a curva de Hadlock (1991) até a $31^{\text {a }}$ semana e a curva de Ramos (1983) a partir da $31^{\text {a }}$ semana.

Das 3093 gestantes que foram selecionadas para o estudo, 933 foram excluídas por se tratar de gestação gemelar, idade inferior a 18 anos, dados incompletos e gestantes com idade entre 30 e 35 anos.

Para a análise estatística, foram utilizados o teste do $\chi^{2}$ e o teste exato de Fisher, quando aplicável. Foi considerado risco a (alfa) menor ou igual a $5 \%$, com intervalo de confiança de $95 \%$.

O estudo foi aprovado pelo Comitê de Ética e Pesquisa do Hospital do Servidor Público Estadual.

\section{Resultados}

Entre as 2160 gestantes que constituíram a população efetivamente em estudo, $1132(52,4 \%)$ tinham de 18 a 29 anos, 792 (36,7\%) de 35 a 39 anos e $236(10,9 \%)$ idade acima de 40 anos. A média e desvio padrão para os grupos entre $18 \mathrm{e}$ 29 anos, 35 e 39 anos e acima de 40 anos foram de 23,1 $( \pm 3,8)$ anos, 36,6 $( \pm 1,4)$ anos e 41,6 $( \pm 1,6)$ anos, respectivamente.

Em relação ao número de primigestas, os grupos entre 35-39 anos e após 40 anos diferiram significantemente $(\mathrm{p}<0,05)$ em comparação com o grupo controle: (18-30 anos) 59,8\%, (35-39 anos) 23\% e (após 40 anos) 20,3\%.

Quanto ao tipo de parto, a cesárea foi o mais freqüente $(46,6 \%)$, tendo sido o fórcipe indicado em $16,7 \%$ dos casos e o parto normal em $36,7 \%$. Tanto o grupo de gestantes entre 35 e 39 anos quanto o grupo acima de 40 anos diferiram significantemente $(\mathrm{p}<0,05)$ do grupo controle em relação à taxa de cesáreas: 55,3 vs $36,7 \%$ e 64,8 vs $36,7 \%$, respectivamente.

Em relação aos recém-nascidos, a idade gestacional média do parto foi de 39,6 semanas $( \pm 15,3$ semanas) para o grupo de gestantes entre 18 e 29 anos, 38,4 ( \pm 4,0 semanas) para o grupo entre 35 e 39 anos e 37,7 ( $\pm 5,2$ semanas) para o grupo acima de 40 anos, sendo significante $(\mathrm{p}<0,05)$ apenas quando comparados o primeiro com o último grupo.

O número de partos prematuros foi de 116 (10,2\%) para o grupo de 18 a 29 anos, $98(12,3 \%)$ para o grupo de 35 a 39 anos e $39(16,5 \%)$ para o grupo com idade acima de 40 anos. Houve diferença significante entre os grupos de 18-29 anos e com mais de 40 anos, quanto à taxa de prematuridade $(\mathrm{p}<0,05)$, como pode ser observado na Tabela 1 .

O peso dos recém-nascidos do grupo de 18 a 29 anos variou entre 520 e 5.075 gramas, com média e desvio padrão de $3.170 \pm 549$ gramas. No grupo de 35 a 39 anos a variação de peso foi entre 560 e 5.185 gramas, com média e desvio padrão de 3.189 \pm 696 gramas. Já entre as pacientes com 
40 anos ou mais as crianças pesaram entre 600 e 4.565 gramas, com média e desvio padrão de $2.989 \pm 600$ gramas, com diferença significante quando comparados os grupos de 18-29 anos e acima de 40 anos $(\mathrm{p}<0,05)$, de acordo com a Tabela 1.

O número de recém-nascidos de baixo peso foi de $88(7,8 \%)$ para o grupo de 18-29 anos, 85 $(10,7 \%)$ para o grupo de $35-39$ anos e de $37(15,7 \%)$ para o grupo acima de 40 anos. Houve diferença significante entre os grupos de 18-29 anos e acima de 40 anos quanto ao número de recém-nascidos de baixo peso $(\mathrm{p}<0,05)$.

Quanto aos casos de RCF, houve maior freqüência no grupo acima de 40 anos (38/236; $16,1 \%$ ), quando comparado ao grupo entre 35-39 anos $(68 / 792 ; 8,6 \%)$ e o grupo entre $18-29$ anos (111/1132; 9,8\%). Houve diferença significante entre os grupos de 18-29 anos e acima de 40 anos quanto ao índice de RCF $(\mathrm{p}<0,05)$ (Tabela 1$)$.
$\mathrm{O}$ índice de Apgar de $1^{\circ}$ minuto menor que sete ocorreu com menor freqüência no grupo controle quando comparado com os grupos 35-39 anos e acima de 40 anos, apresentando diferença significante $(\mathrm{p}<0,05)$ entre os grupos de $18-29$ e acima de 40 anos: 18-29 anos (107/1132; 9,4\%), $35-39$ anos $(98 / 792 ; 12,3 \%)$ e acima de 40 anos (35/236; 14,8\%). Porém, os mesmos achados não se repetiram em relação ao índice de Apgar de $5^{\circ}$ minuto menor que sete, que não mostrou diferença significante entre os grupos $(\mathrm{p}=0,06): 18-29$ anos (15/1132; 1,3\%), 35-39 anos (6/792; 0,8\%) e acima de 40 anos $(3 / 236 ; 1,3 \%)$.

Ocorreram 11 casos de óbitos fetais $(0,98 \%)$ no grupo de $18-29$ anos, 8 casos $(1,0 \%)$ no grupo de $35-39$ anos e 13 casos (5,5\%) no grupo acima de 40 anos. Constatou-se diferença significante entre os grupos de 18-29 anos e acima de 40 anos, quanto à ocorrência de óbito fetal $(\mathrm{p}<0,05)$ (Tabela 1$)$.

Tabela 1 - Resultados perinatais de acordo com a faixa etária das mães.

\section{Grupos (idade/anos)}

\begin{tabular}{lcccccc} 
& $\mathbf{1 8 - 2 9}$ & $\%$ & $\mathbf{3 5 - 3 9}$ & $\mathbf{\%}$ & $\mathbf{> 4 0}$ & $\%$ \\
\hline Resultados perinatais & $(\mathrm{n}=1132)$ & & $(\mathrm{n}=792)$ & & $(\mathrm{n}=236)$ & \\
Prematuridade & 116 & 10,2 & 98 & 12,3 & 39 & 16,5 \\
Baixo peso ao nascer & 88 & 7,8 & 85 & 10,7 & 37 & 15,7 \\
Restrição do crescimento fetal & 111 & 9,8 & 68 & 8,6 & 38 & 16,1 \\
Óbito fetal & 11 & 0,98 & 8 & 1,0 & 13 & 5,5 \\
\hline
\end{tabular}

\section{Discussão}

A freqüência de $22,5 \%$ de gestantes tardias em nossa casuística foi maior que a encontrada na literatura, na qual os índices não costumam ser superiores a $10 \%{ }^{2}$. Pode-se justificar este achado por se tratar eminentemente de uma população de funcionárias públicas, com nível socioeconômico e cultural diferenciado que optam, muitas vezes, por adiar suas gestações até a obtenção de certo grau de estabilidade econômica e profissional.

Entre as com mais de 35 anos, observou-se, quanto à via de parto, índice de cesáreas mais alto em relação ao grupo controle. Algumas condições maternas associadas ao possivel comprometimento fetal costumam ser mais freqüentes e mais graves nesta faixa etária e, portanto, elevam o número de cesáreas por indicação fetal ${ }^{4,7,16,17}$. As distocias do trabalho de parto também tendem a ocorrer mais vezes entre as gestantes tardias e também acabam por levar a maior número de par- tos abdominais ${ }^{4,27}$. Finalmente, é possivel que a idade materna mais avançada possa fazer com que muitos médicos tornem-se ansiosos quanto ao bem estar fetal. Isso talvez decorra dos preconceitos sobre os riscos relacionados com a idade ou de uma legítima intenção de assegurar um prognóstico adequado para a gestante com mais idade, especialmente se submetida a tratamento para infertilidade. Edge e Laros $^{9}$ mostraram que a ansiedade dos médicos em relação a gestantes em fase de decréscimo de fertilidade, freqüentemente após uso de técnicas de reprodução assistida, induz a escolha da via de parto, aumentando o número de cesáreas sem indicação obstétrica.

A alta taxa de prematuridade acima de 40 anos, diferindo significantemente quando comparada ao grupo controle ( 18 a 29 anos), está de acordo com os trabalhos de Edge e Laros ${ }^{9}$, Dildy et al. ${ }^{12}$, Jolly et al. ${ }^{21}$ e outros como os de Ziadeh e Yahaya ${ }^{17}$, Wong e $\mathrm{Ho}^{3}$, Romero-Maldonado et al. ${ }^{11}$ e Azevedo et al. ${ }^{23}$, não sendo confirmados pelos estudos de Chan e $\mathrm{LaO}^{4}$. 
Eisenberg e Schenker ${ }^{10}$ mostraram taxa de mortalidade perinatal aumentada em gestantes com mais de 40 anos, o que está de acordo com os resultados deste estudo. Os principais fatores que contribuem para o aumento da mortalidade perinatal após os 35 anos, além da paridade e do nível socioeconômico, são a prematuridade, a restrição ao crescimento fetal, as anomalias congênitas, os traumas de parto, a anóxia e as infecções ${ }^{21}$

Em nosso estudo, a incidência de baixo peso ao nascer foi superior em gestantes tardias, o que está de acordo com Ziadeh ${ }^{18}$ e Azevedo et al. ${ }^{23}$, e é um dos fatores implicados nos aumentos dos índices de mortalidade perinatal. Verificou-se predomínio de RCF no grupo de pacientes com mais de 40 anos, o que está de acordo com os estudos de Edge e Laros ${ }^{9}$, Eisenberg e Schenker ${ }^{10}$, Dildy et al. ${ }^{12}$ e Yuan et $\mathrm{al}^{13}$.

Em relação ao índice de Apgar, observou-se diferença significante para valores menores de sete quando comparamos o grupo de 18-29 anos com ode pacientes com idade superior a 40 anos. Este achado foi diferente do encontrado nos estudos de Prysac et al. ${ }^{1}$ e Edge e Laros ${ }^{9}$, que não mostraram diferença significativa nos índices de Apgar de primeiro e quinto minuto em gestantes acima de 35 anos.

Quanto à ocorrência de óbito fetal, nosso estudo constatou significante diferença nas pacientes com idade superior a 40 anos em relação ao grupo controle de 18-29 anos, mostrando incidência cerca de cinco vezes maior. Essas taxas foram superiores às da literatura, que evidenciou valores máximos de 2,4 vezes maior de casos nas pacientes com idade superior a 40 anos em relação ao controle . $^{2}$

Não está claro se uma mulher com mais de 35 anos, gozando de boa saúde, sem história de infertilidade, não fumante e com características sociodemográficas favoráveis apresenta risco gestacional mais elevado. Certamente, esta gestante corre risco menor em termos de resultados gestacionais adversos do que outra que apresente uma doença clínica, que conte com história de infertilidade e problemas ginecológicos, que fume ou que faça uso de drogas. Mesmo assim, a idade avançada em si eleva os riscos até mesmo de uma mulher saudável, principalmente em gestantes acima de $40 \operatorname{anos}^{25,26}$.

Pelo que foi exposto, fica evidente a necessidade de mais estudos sobre esse assunto e de seguimento pré-natal especializado para as gestantes com idade superior a 40 anos. Desta forma, poderemos atuar sobre fatores de risco e intercorrências clínicas que possam advir, minimizando as complicações e melhorando o prognóstico destas gestantes.

\section{ABSTRACT}

Purpose: to evaluate perinatal results in pregnant women over 35 years old and to check differences between two groups: 35 to 39-year-old women and women older than 40.

Methods: a retrospective survey was made during the period between January/2000 and July/2003, through the analysis of obstetric charts of 3,093 pregnant women who delivered in the "Hospital do Servidor Público Estadual - Francisco Morato de Oliveira”, excluding 933 patients. The patients were divided into 3 groups: 18 to 29 years old (control group), 35 to 39 years old, and over 40 years old. Data collection was done with standardized forms, and the data were transferred to an electronic spreadsheet (Excel-Microsoft Office 2000). Statistical analysis was performed using the $\chi^{2}$ test and the Fisher test. The alpha risk was less or equal to $5 \%$ and the confidence interval $95 \%$.

Results: cesarean section was the most used method not only in the 35 to 39-year-old group $(438 / 792 ; 55.3 \%)$ but also in the group of women over 40 (153/236; 64.8\%). The rates of prematurity (39/236; 16.5\%), low weight (37/236; 15.7\%), and restriction of fetal growth $(38 / 236 ; 16.1 \%)$ were significantly higher in the group of women over 40, when compared to the other groups. Concerning fetal death, a five times higher incidence was observed in the group over 40 years old, as compared to the other groups, a statistically significant difference.

Conclusion: the only difference between the 35 to 39-yearold group and the control group was the cesarean section rate. This allows us to suggest a differentiated prenatal attendance for pregnant women over 40.

KEYWORDS: Maternal age. Normal pregnancy. Prematurity. Fetal growth restriction.

\section{Referências}

1. Prysak M, Lorenz RP, Kisly A. Pregnancy outcome in nulliparous women 35 years and older. Obstet Gynecol 1995; 85:65-70.

2. Sauer MV, Paulson RJ, Lobo RA. Oocyte donation to women of advanced reproductive age: pregnancy results and obstetrical outcomes in patients 45 years and older. Hum Reprod 1996; 11:2540-3.

3. Wong SF, Ho LC. Labour outcome of low-risk multiparas of 40 years and older. A case-control study. Aust N Z J Obstet Gynaecol 1998; 38:388-90.

4. Chan BC, Lao TT. Influence of parity on the obstetric performance of mothers aged 40 years and above. Hum Reprod 1999; 14:833-7.

5. Dulitzki M, Soriano D, Schiff E, Chetrit A, Mashiach $\mathrm{S}$, Seidman DS. Effect of very advanced maternal 
age on pregnancy outcome and rate of cesarean delivery. Obstet Gynecol 1998; 92:935-9.

6. Adashek JA, Peaceman AM, Lopez-Zeno JA, Minogue JP, Socol ML. Factors contributing to the increased cesarean birth rate in older parturient women. Am J Obstet Gynecol 1993; 169:936-40.

7. Bianco A, Stone J, Lynch L, Lapinski R, Berkowitz G, Berkowitz RL. Pregnancy outcome at age 40 and older. Obstet Gynecol 1996; 87:917-22.

8. Donoso Siña E, Villarroel del Pino L. Reproductive risk of women over 40 years old. Rev Med Chile 2003; 131:55-9.

9. Edge V, Laros RK Jr. Pregnancy outcome in nulliparous women aged 35 or older. Am J Obstet Gynecol 1993; 168:1881-4.

10.Eisenberg VH, Schenker JG. Pregnancy in the older woman: scientific and ethical aspects. Int $\mathrm{J}$ Gynaecol Obstet 1996; 56:163-9.

11.Romero-Maldonado S, Quezada-Salazar CA, Lopez Barrera MD, Arroyo Cabrales LM. Efecto de riesgo en el hijo de madre con edad avanzada (estudio de casos y controles). Ginecol Obstet Mex 2002; 70:295-302.

12.Dildy GA, Jackson GM, Fowers GK, Oshiro BT, Varner MW, Clark SL. Very advanced maternal age: pregnancy after age 45. Am J Obstet Gynecol 1996; 175:668-74.

13.Yuan W, Steffensen FH, Nielsen GL, Moller M, Olsen J, Sorensen HT. A population-based cohort study of birth and neonatal outcome in older primipara. Int J Gynaecol Obstet 1999; 68:113-8.

14.Ecker JL, Chen KT, Cohen AP, Riley LE, Lieberman ES. Increased risk of cesarean delivery with advancing maternal age: indications and associated factors in nulliparous women. Am J Obstet Gynecol 2001; 185:883-7.

15.Kozinszky Z, Orvos H, Katona M, Zoboki T, Pal A, Kovacs L. Perinatal outcome of induced and spontaneous pregnancies of primiparous women aged 35 or over. Int J Gynaecol Obstet 2002; 76:23-6.

16.Gilbert WM, Nesbitt TS, Danielsen B. Childbearing beyond age 40: pregnancy outcome in 24,032 cases.
Obstet Gynecol 1999; 93:9-14.

17.Ziadeh S, Yahaya A. Pregnancy outcome at age 40 and older. Arch Gynecol Obstet 2001; 265:30-3.

18.Ziadeh SM. Maternal and perinatal outcome in nulliparous women aged 35 and older. Gynecol Obstet Invest 2002; 54:6-10.

19.Sheiner E, Shoham-Vardi I, Hershkovitz R, Katz M, Mazor M. Infertility treatment is an independent risk factor for cesarean section among nulliparous women aged 40 and above. Am J Obstet Gynecol 2001; 185:888-91.

20.Scholz HS, Haas J, Petru E. Do primiparas aged 40 years or older carry an increased obstetric risk? Prev Med 1999; 29:263-6.

21.Jolly M, Sebire N, Harris J, Robinson S, Regan L. The risks associated with pregnancy in women aged 35 years or older. Hum Reprod 2000; 15:2433-7.

22.Vega CEP, Marcus PAF, Pazero LC, Boyaciyan K, Barbosa SA. Estudo da mortalidade materna no município de São Paulo durante o ano de 1997. Rev Ginecol Obstet 2001; 12:187-97.

23.Azevedo GD, Freitas Júnior RAO, Freitas AKMSO, Araújo ACPF, Soares EMM, Maranhão TMO. Efeito da idade materna sobre os resultados perinatais. Rev Bras Ginecol Obstet 2002; 24:181-5.

24.Laurenti R, Buchalla CM. Estudo da morbidade e da mortalidade perinatal em maternidades. IIMortalidade perinatal segundo peso ao nascer, idade materna, assistência pré-natal e hábito de fumar da mãe. Rev Saúde Pública 1985; 19:225-32.

25.Romero Gutierrez G, Bribiesca Lopez JA, Ramos Palma S, Bravo Aguirre DE. Morbilidad y mortalidad materno fetal en embarazadas de edad avanzada. Ginecol Obstet Mex 1999; 67:239-45.

26.Vercellini P, Zuliani G, Rognoni MT, Trespidi L, Oldani S, Cardinale A. Pregnancy at forty and over: a case-control study. Eur J Obstet Gynecol Reprod Biol 1993; 48:191-5.

27.Halpern R, Schaefer ES, Pereira AS, Arnt EM, Bezerra JPV, Pinto LS. Fatores de risco para baixo peso ao nascer em uma comunidade rural do sul do Brasil. J Pediatr (RJ) 1996; 72:369-73. 